\title{
Frequency of Positive Studies Among Fixed and Flexible Dose Antidepressant Clinical Trials: An Analysis of the Food and Drug Administraton Summary Basis of Approval Reports
}

\author{
Arif Khan*,1,2, Shirin R. Khan', Gary Walens ${ }^{3}$, Russell Kolts ${ }^{4}$ and Earl L. Giller ${ }^{3,5}$ \\ 'Northwest Clinical Research Center, Bellevue, WA, USA; ${ }^{2}$ Department of Psychiatry and Behavioral Sciences, Duke University Medical Center, \\ Durham, NC, USA; ${ }^{3}$ Pfizer Global Research and Development, New London, CT, USA; ${ }^{4}$ Department of Psychology, Eastern Washington \\ University, Cheney, WA, USA; ${ }^{5}$ Department of Psychiatry, Yale School of Medicine, New Haven, CT, USA
}

\begin{abstract}
The assumption that the design of an antidepressant clinical trial affects the outcome of that trial is based on sparse data. We sought to examine if the dosing schedule, either a fixed dose or a flexible dose type, in an antidepressant clinical trial affects the frequency with which antidepressants show statistical superiority over placebo. Randomized, placebo-controlled clinical trials of nine antidepressants approved by the Food and Drug Administration between 1985 and 2000 were reviewed. These trials comprised 93।3 depressed patients who participated in $5 \mathrm{I}$ antidepressant clinical trials consisting of 92 treatment arms with eventual approved doses. In the flexible dose trials, 59.6\% (34/57) of the antidepressant treatment arms were statistically significant compared to placebo, whereas in the fixed dose trials only $31.4 \%(1 / 135)$ of the antidepressant treatment arms were statistically significant compared to placebo $\left(\chi^{2}=6.9, \mathrm{df}=1\right.$, $p<0.0 \mathrm{I})$. These data suggest that the antidepressant dose schedule may influence trial outcome due in part to a significantly lower magnitude of symptom reduction with placebo in flexible dose trials $(F=4.08, d f=1,48, p<0.05)$ compared to fixed dose trials. Symptom reduction was similar with antidepressants in the flexible and fixed dose trials. Further, the primary function of finding a doseresponse relationship was not found among the fixed dose studies.

Neuropsychopharmacology (2003) 28, 552-557. doi: I0.I 038/sj.npp. 1300059
\end{abstract}

Keywords: antidepressants; clinical trials; placebo; fixed dose; flexible dose; meta-analysis

\section{INTRODUCTION}

Randomized, double-blind, placebo-controlled clinical trials have become the essential tool to evaluate the safety and efficacy of a putative medical treatment. However, clinical trials of psychotherapeutic agents are plagued with high failure rates, and results from individual trials are often difficult to interpret. Variables that are hard to alter, such as characteristics of the disorder and medication under study, can contribute to the increased probability of a failed trial. Under more control, however, is the design of the clinical trial.

A clinical assumption, in antidepressant clinical trials, is that a flexible dose schedule retains patients and optimizes response better than a fixed dose schedule. However, the scientific literature on antidepressant clinical trials contains few studies assessing either the outcome or advantages and

*Correspondence: Dr A Khan, 1900 166th AVE NE \# I 12, Bellevue, WA 98004, USA, Tel: +I 425452 0404, Fax: + I 425453 103,

E-mail: akhan@nwcrc.net

Received 4 June 2002; revised 22 August 2002; accepted 10 September 2002

Online publication: 16 September 2002 at http://www.acnp.org/ citations/Npp091702382 disadvantages of fixed dose studies compared to flexible dose studies. Much of previous research relating to antidepressant dosing has involved tricyclic antidepressants (TCA), and the major aim has been to find the lowest optimal dose of the antidepressant with the best adverse event profile (Gram 1990; Benkert et al, 1996; Bollini et al, 1999) to minimize side effects. An intriguing suggestion that lower doses may work as well or better when using SSRIs has not been further explored (Schatzberg, 1991).

Thus, we sought to examine the degree to which flexible dose and fixed dose, double-blind, randomized, placebocontrolled antidepressant clinical trials are positive using a meta-analytic approach. To address the lack of information regarding fixed dose trials compared to flexible dose trials, we examined the Food and Drug Administration (FDA) Summary for Basis of Approval (SBA) reports, accessible via the Freedom of Information Act (US Congress, 1996), for recently approved antidepressants to characterize the frequency of positive fixed dose and positive flexible dose trials.

Specifically, we undertook an exploratory analysis to determine whether a fixed dose or a flexible dose schedule impacts antidepressant clinical trial outcome. This analysis includes the results from 51 antidepressant clinical trials 
that were submitted to the FDA by the sponsoring pharmaceutical companies to gain drug approval. We sought to assess: (1) the relationship between placebo response magnitude, as measured by change on mean total HAM-D score (Hamilton Depression Rating Scale), and antidepressant response; (2) the frequency of statistical superiority of an antidepressant under investigation or an active comparator over placebo; and (3) differences in statistical superiority over placebo between clinical trials that incorporated a fixed dose and those with a flexible dose.

As the effects of dosing may be influenced by other factors in clinical trial design, we assessed several of these potential confounding factors as follows: (1) presence of active comparator in the trials, (2) duration of the trial, (3) sample size for each treatment arm, (4) number of treatment arms for each trial, (5) trial completion rates, and (6) whether the aim of showing a dose-response relationship was achieved in fixed dose trials.

\section{MATERIALS AND METHODS}

We obtained the FDA clinical trial database (statistical and clinical reports) for all antidepressants approved in the United States from 1 January 1985 to 31 December 1998. Specifically, under the Freedom of Information Act (US Congress, 1996) we obtained public domain data on FDAreviewed studies for fluoxetine hydrochloride (Prozac), sertraline hydrochloride (Zoloft), paroxetine hydrochloride (Paxil), nefazodone hydrochloride (Serzone), mirtazapine (Remeron), bupropion hydrochloride (Wellbutrin SR), venlafaxine hydrochloride (Effexor), venlafaxine hydrochloride ER (Effexor XR), and citalopram hydrobromide (Celexa) by a specific request to the FDA Freedom of Information Staff (Rm 12A-16, 5600 Fishers Lane, Rockville, MD 20857). Images of the summary basis of approval documents were sent by mail on microfiche.

From these nine research programs, the FDA considered 56 clinical trials to be pivotal; of these, we excluded five trials from our analysis. Three trials were excluded because of insufficient data, one because it focused on relapse prevention rather than acute treatment response, and one because the investigational treatment did not evaluate a dose approved by the FDA for marketing. Of the 51 clinical trials reviewed in this analysis, there were 79 treatment arms for the antidepressant under investigation (hereafter referred to as 'new antidepressants'); of these, 10 treatment arms were excluded as they evaluated doses that are not approved for treatment. Thus, all arms included for analysis used doses considered within the therapeutic range by the approved label. Additionally, an active comparator (imipramine, amitriptyline, trazodone, sertraline, or venlafaxine) was used in 23 of these 51 clinical trials $(2 / 15$ in the fixed trials, $21 / 36$ in the flexible trials). Thus, a total of 92 active treatment arms were evaluated. We categorized the nature of dosing, fixed or flexible, based on data in SBA reports.

In the 51 clinical trials (for more specific study information see Khan et al, 2000, 2001), 9313 patients were included in the Intent-to-Treat analysis; 3335 assigned to placebo, 4676 assigned to a new antidepressant, and 1502 assigned to an active comparator.
Each of the studies used the following standard subject inclusion and exclusion criteria; (1) 18 years of age or older; (2) patients in each trial had a diagnosis of major depression of moderate to severe degree, according to DSM-III, DSM-III-R, Research Diagnostic Criteria, Newcastle Endogenous/Reactive Depression Rating Scale, or DSM-IV; (3) signed informed consent form (witnessed); (4) a minimum score at screen evaluation of $14,18,20$, or 22 on total HAM-D 17, 18 on HAM-D 18, 18 or 20 on HAM-D 21, 25 on HAM-D 24, or 22 or 25 on MADRS; (5) 4-10 days of placebo run-in; (6) less than a $20 \%$ decrease in HAM-D score between screen and baseline; (7) not actively suicidal or posing a serious suicidal risk; and (8) no significant concurrent or previous other psychiatric illnesses.

Each study has the following design features: (1) primary efficacy measures included HAM-D; (2) mean total raw scores and change in scores on the HAM-D were available for calculations and comparisons; and (3) depressed patients underwent evaluation at weekly intervals during the first month and at least biweekly after 4 weeks.

Change from baseline in mean total HAM-D score is typically specified as the primary outcome measure in antidepressant clinical trials. However, because of the heterogeneity of these trials, percent change in mean total HAM-D scores was used as an index of symptom reduction because it accounts for differences in baseline severity. When the data on absolute changes were analyzed, the results were very similar to those presented here. We were unable to calculate effect sizes for many of the trials as standard deviation or standard error was not available for each trial.

We based assessments on whether or not each arm of a clinical trial was considered positive (new antidepressant or active comparator superior to placebo) based on a nominal $p$ value $\leqslant 0.05$ as assessed by the FDA staff. Hence the $p$ values for each of the arms of the multiple dose arm studies were not corrected for multiple comparisons. Response rates (percentage of patients showing therapeutic response, usually a $50 \%$ reduction in HAM-D score) were not available in the FDA reports.

Trials with a fixed dose of the antidepressant under investigation were considered separately from trials with a flexible dose of the antidepressant under investigation (Tables 1 and 2). As the overall design of these trials was similar, $\chi^{2}$ test was conducted to assess the frequency with which a treatment arm with a flexible dose of the new antidepressant was statistically significant compared to a treatment arm with a fixed dose of the new antidepressant, both compared to placebo.

Since flexible dose trials had a higher ratio of active comparator treatment arms, we assessed the significance of the presence of active comparator groups.

Other characteristics that may affect trial outcome were duration of the trial and the sample size in each trial. We conducted analyses examining the relationship between these two factors and outcome with placebo and antidepressants in both the flexible and fixed dose studies. Additional factors such as number of treatment arms and number of patients completing the trials were assessed between flexible and fixed dose studies. Lastly, we attempted to ascertain if the primary function of the fixed dose trials, ie establishing a dose-response relationship, was 
Table I Magnitude of Symptom Reduction among Depressed Patients Assigned to Placebo or Antidepressants in 36 Flexible Dose Pivotal Clinical Trials ${ }^{\mathrm{a}}$

\begin{tabular}{|c|c|c|c|c|c|c|}
\hline \multirow[b]{2}{*}{$\begin{array}{l}\text { New antidepressant: } \\
\text { protocol }\end{array}$} & \multirow[b]{2}{*}{$\begin{array}{l}\text { Duration of } \\
\text { trial (weeks) }\end{array}$} & \multirow{2}{*}{$\begin{array}{l}\text { Placebo } \\
\text { \% symptom } \\
\text { reduction }\end{array}$} & \multicolumn{2}{|c|}{ New antidepressant } & \multicolumn{2}{|c|}{ Active comparator } \\
\hline & & & $\begin{array}{l}\text { \% symptom } \\
\text { reduction }\end{array}$ & $\begin{array}{l}\text { Dose } \\
\left(\mathbf{m g} / \text { day }^{\mathrm{b}}\right)\end{array}$ & $\begin{array}{l}\text { \% symptom } \\
\text { reduction }\end{array}$ & $\begin{array}{l}\text { Dose } \\
\left(\mathbf{m g} / \text { day }^{\mathrm{b}}\right)\end{array}$ \\
\hline I. Paroxetine: 03-006 & 6 & 10.5 & 30.6 & $10-50$ & 27.2 & $10-50^{c}$ \\
\hline 2. Paroxetine: 03-005 & 6 & 15.3 & 38.3 & $10-50$ & 56.9 & $65-275^{c}$ \\
\hline 3. Mirtazapine: 003-020/3220 & 6 & 16.3 & 38.1 & $5-35$ & 39.4 & $40-\mathbf{2 8 0}$ \\
\hline 4. Venlafaxine: $600 \mathrm{~A}-206$ & 4 & 16.8 & 50.4 & $150-375$ & - & \\
\hline 5. Fluoxetine: 19 & 5 & 19.5 & 43.7 & $\geqslant 40$ & - & \\
\hline 6. Paroxetine: $03-001$ & 4 & 20.6 & 39.4 & $10-50$ & 29.0 & $65-275^{d}$ \\
\hline 7. Mirtazapine: 003-002 & 6 & 21.9 & 48.3 & $5-35$ & - & \\
\hline 8. Paroxetine: $02-002$ & 6 & 23.3 & 43.6 & $10-50$ & - & \\
\hline 9. Citalopram: $86|4|$ & 6 & 23.8 & 28.4 & $20-80$ & - & \\
\hline 10. Paroxetine: 03-002 & 4 & 24.2 & 32.1 & $10-50$ & 32.0 & $65-275^{c}$ \\
\hline I।. Paroxetine: 03-004 & 4 & 24.8 & 37.7 & $10-50$ & 21.9 & $65-275^{c}$ \\
\hline 12. Paroxetine: $02-00 \mid$ & 6 & 26.3 & 46.2 & $10-50$ & - & \\
\hline 13. Paroxetine: 02-003 & 6 & 27.7 & 35.3 & $10-50$ & - & \\
\hline |4. Mirtazapine: 003-024/3220 & 6 & 27.8 & 44.0 & $5-35$ & 50.4 & $40-280^{d}$ \\
\hline 15. Paroxetine: 02-004 & 4 & 27.8 & 43.9 & $10-50$ & - & \\
\hline 16. Mirtazapine: 003-023/3220 & 6 & 28.1 & 40.2 & $5-35$ & 31.7 & $40-280^{\mathrm{e}}$ \\
\hline 17. Citalopram: $85 \mathrm{~A}$ & 4 & 28.5 & 38.5 & $20-80$ & - & \\
\hline 18. Venlafaxine ER: 209 & 8 & 28.8 & 47.8 & $75-225$ & - & \\
\hline 19. Mirtazapine: 003-022/3220 & 6 & 28.8 & 48.8 & $10-35$ & 44.1 & $40-280^{d}$ \\
\hline 20. Fluoxetine: 27 & 6 & 29.8 & 40.0 & $\geqslant 40$ & 42.6 & $\geqslant 150^{c}$ \\
\hline 2I. Nefazadone: CNI04-005 & 8 & 34.0 & 49.2 & $100-600$ & 42.0 & $50-300^{c}$ \\
\hline 22. Paroxetine: 03-003 & 4 & 34.1 & 36.2 & $10-50$ & 29.7 & $65-275^{c}$ \\
\hline 23. Fluoxetine: 25 & 5 & 34.1 & 27.5 & $\geqslant 40$ & - & \\
\hline 24. Mirtazapine: 003-003 & 6 & 34.5 & 40.9 & $10-35$ & - & \\
\hline 25. Mirtazapine: 84023 & 6 & 34.6 & 42.6 & $15-50$ & - & \\
\hline 26. Sertraline: 104 & 8 & 35.0 & 50.2 & $50-200$ & 54.3 & $50-150^{d}$ \\
\hline 27. Sertraline: 315 & 8 & 35.1 & 38.5 & $50-200$ & 49.4 & $50-150^{d}$ \\
\hline 28. Venlafaxine ER: 208 & 12 & 35.4 & 61.1 & $75-150$ & 52.1 & $75-150^{f}$ \\
\hline 29. Nefazadone: CNI04-002 & 6 & 35.5 & 46.4 & $50-300$ & 58.7 & $50-300^{c}$ \\
\hline 30. Venlafaxine: $600 \mathrm{~A}-302$ & 6 & 36.5 & 47.6 & $75-200$ & 42.9 & $150-400^{\circ}$ \\
\hline 31. Nefazadone: CNI04-006 & 8 & 37.4 & 42.6 & $100-600$ & 46.4 & $50-300^{c}$ \\
\hline 32. Paroxetine: $01-001$ & 6 & 38.3 & 48.2 & $10-50$ & - & \\
\hline 33. Venlafaxine: $600 \mathrm{~A}-30$ I & 6 & 38.6 & 54.7 & $75-225$ & 43.4 & $75-225^{c}$ \\
\hline 34. Mirtazapine: 003-021/3220 & 6 & 38.9 & 48.3 & $10-35$ & 54.4 & $40-280$ \\
\hline 35. Venlafaxine: $600 \mathrm{~A}-303$ & 6 & 40.2 & 42.8 & $75-225$ & 40.9 & $75-225^{c}$ \\
\hline 36. Mirtazapine: 85027 & 5 & 41.6 & 50.8 & $20-60$ & - & \\
\hline Mean values \pm SD & & $29.3 \pm 7.8$ & $42.9 \pm 7.2$ & & $42.4 \pm 10.5$ & \\
\hline
\end{tabular}

${ }^{a}$ Symptom reduction is measured by change in mean total HAM-D score from baseline to LOCF. The clinical trials are listed in ascending order of symptom reduction with placebo. Bold type indicates statistical superiority over placebo $(p \leqslant 0.05)$. Ellipses indicate not applicable. ${ }^{b}$ Drug dosages are titrated (range). Only treatment arms with approved dosing for market are included. Imipramine. ${ }^{\mathrm{d}}$ Amitriptyline. ${ }^{\mathrm{e}}$ Trazodone. ${ }^{\mathrm{f}}$ Venlafaxine.

achieved after excluding doses not considered to be therapeutic by the FDA.

\section{RESULTS}

In the flexible dose trials (Table 1), placebo-treated patients showed a mean decrease in total percent symptom reduction in HAMD score of $29.3 \%$; patients receiving a new antidepressant, 42.9\%; and patients receiving an active comparator, $42.4 \%$. A total of $58 \%(21 / 36)$ of the new antidepressant treatment arms were superior to placebo, whereas $61.9 \%(13 / 21)$ of the active comparator treatment arms were superior to placebo.

In the fixed dose trials (Table 2), placebo-treated patients showed a mean decrease in total percent symptom reduction in HAMD score of $35.8 \%$; patients receiving a new antidepressant, $42.2 \%$; and patients receiving an active comparator, $42.6 \%$. A total of $30 \%(10 / 33)$ of the new antidepressant treatment arms were superior to placebo, whereas $50.0 \%(1 / 2)$ of the active comparator treatment arms were superior to placebo.

When all 92 new antidepressant clinical arms (including both new antidepressants and active comparators) were considered for analysis, the antidepressant treatment arms from flexible dose trials were more likely to show statistically significant superiority over placebo (34/57, $59.6 \%)$ compared to the antidepressant treatment arms from fixed dose trials $(11 / 35,31.4 \%)\left(\chi^{2}=6.91, \mathrm{df}=1\right.$, $p \leqslant 0.01$ ).

The presence of an active comparator in the flexible dose trials did not seem to affect trial outcome. In the flexible dose trials without an active comparator $(n=13)$, placebo response was $28.5 \%$ and new antidepressant response was $42.4 \%$, with $53.3 \%(8 / 13)$ of the new antidepressant 
Table 2 Magnitude of Symptom Reduction among Depressed Patients Assigned to Placebo or Antidepressants in 15 Fixed Dose Pivotal Clinical Trials ${ }^{\mathrm{a}}$

\begin{tabular}{|c|c|c|c|c|c|c|}
\hline \multirow[b]{2}{*}{$\begin{array}{l}\text { New antidepressant: } \\
\text { protocol }\end{array}$} & \multirow[b]{2}{*}{$\begin{array}{l}\text { Duration of } \\
\text { trial (weeks) }\end{array}$} & \multirow{2}{*}{$\begin{array}{l}\text { Placebo } \\
\text { \% symptom } \\
\text { reduction }\end{array}$} & \multicolumn{2}{|c|}{ New antidepressant } & \multicolumn{2}{|c|}{ Active comparator } \\
\hline & & & $\begin{array}{l}\% \text { symptom } \\
\text { reduction }\end{array}$ & $\begin{array}{l}\text { Dose } \\
\left(\mathrm{mg} / \text { day }^{\mathrm{b}}\right)\end{array}$ & $\begin{array}{l}\text { \% symptom } \\
\text { reduction }\end{array}$ & $\begin{array}{l}\text { Dose } \\
\left(\mathrm{mg} / \text { day }^{\mathrm{b}}\right)\end{array}$ \\
\hline \multirow[t]{3}{*}{ I. Fluoxetine: 62} & 6 & 23.5 & 39.7 & 20 & - & \\
\hline & & & 39.8 & 40 & & \\
\hline & & & 29.8 & 60 & & \\
\hline \multirow[t]{2}{*}{ 2. Nefazadone: 03AOA-003 } & 6 & 26.3 & 32.5 & $50-250$ & 41.9 & $\mathbf{5 0}-\mathbf{2 5 0} 0^{\circ}$ \\
\hline & & & 43.0 & 500 & & \\
\hline \multirow[t]{3}{*}{ 3. Venlafaxine: $600 \mathrm{~A}-203$} & 6 & 26.5 & 42.7 & 75 & - & \\
\hline & & & 45.8 & $150-225$ & & \\
\hline & & & 42.2 & $300-375$ & & \\
\hline \multirow[t]{3}{*}{ 4. Sertraline: 103} & 6 & 30.0 & 42.7 & 50 & - & \\
\hline & & & 39.4 & 100 & & \\
\hline & & & 35.8 & 200 & & \\
\hline 5. Bupropion: 203 & 8 & 34.9 & 43.6 & 300 & - & \\
\hline \multirow[t]{2}{*}{ 6. Bupropion: 205} & 8 & 35.5 & 38.1 & 300 & - & \\
\hline & & & 38.4 & 400 & & \\
\hline \multirow[t]{2}{*}{ 7. Nefazadone: 030A2-0007 } & 6 & 37.1 & 51.0 & 200 & - & \\
\hline & & & 42.1 & 300 & & \\
\hline \multirow[t]{3}{*}{ 8. Mirtazapine: 003-008 } & 6 & 37.2 & 29.2 & 15 & - & \\
\hline & & & 26.7 & 30 & & \\
\hline & & & 32.0 & 60 & & \\
\hline \multirow[t]{2}{*}{ 9. Venlafaxine: $600 \mathrm{~A}-313$} & 6 & 37.4 & 42.6 & $50-75$ & - & \\
\hline & & & 46.1 & $150-200$ & & \\
\hline \multirow[t]{3}{*}{ 10. Citalopram: 91206 } & 6 & 37.8 & 40.6 & 20 & - & \\
\hline & & & $\mathbf{5 0 . 0}$ & 40 & & \\
\hline & & & 49.4 & 60 & & \\
\hline \multirow[t]{2}{*}{ I I. Nefazadone: 03AOA-004A } & 6 & 37.9 & 35.8 & $150-300$ & - & \\
\hline & & & 40.3 & $300-600$ & & \\
\hline \multirow[t]{2}{*}{ 12. Nefazadone: 03AOA-004B } & 6 & 38.0 & 40.2 & $100-300$ & - & \\
\hline & & & 50.0 & $300-600$ & & \\
\hline 13. Bupropion: 212 & 8 & 41.0 & 45.5 & 300 & & \\
\hline 14. Citalopram: 89303 & 6 & 44.7 & 45.7 & 40 & & \\
\hline \multirow[t]{2}{*}{ 15. Venlafaxine ER: 367} & 8 & 49.2 & 57.8 & 20 & 43.3 & $20^{d}$ \\
\hline & & & 58.9 & 75 & & \\
\hline Mean values $\pm S D$ & $35.8 \pm 6.9$ & $42.2 \pm 7.6$ & 53.9 & 150 & $42.6 \pm 1.0$ & \\
\hline
\end{tabular}

aSymptom reduction is measured by change in mean total HAM-D score from baseline to LOCF. The clinical trials are listed in ascending order of symptom reduction with placebo. Bold type indicates statistical superiority over placebo $(p \leq 0.05)$. Ellipses indicate not applicable. ${ }^{b}$ Drug dosages are fixed (maximum daily dose). Only treatment arms with approved dosing for market are included. Imipramine. ${ }^{d}$ Sertraline.

treatment arms statistically superior to placebo. In the flexible dose trials with an active comparator $(n=21)$, placebo response was $29.9 \%$ and new antidepressant response was $43.2 \%$, with $61.9 \%(13 / 21)$ of the new antidepressant treatment arms statistically superior to placebo. There was no significant difference between the number of positive new antidepressant treatment arms flexible dose trials that had an active comparator and those that did not $\left(\chi^{2}=0.07, \mathrm{df}=1, p=\mathrm{ns}\right)$. Similar results were seen for the two fixed dose trials with an active comparator.

Duration of trial was not statistically significantly different between flexible dose trials $(6.0 \pm$ SD 1.6 weeks) and fixed dose trials $(6.5 \pm \mathrm{SD} 0.9$ weeks, $t=1.29, \mathrm{df}=49$, $p=\mathrm{ns})$. However, the sample size per treatment was statistically significantly larger in fixed dose trials $(81 \pm$ SD 33) compared to flexible dose trials $(59 \pm \mathrm{SD}$ $31.5, t=2.38, \mathrm{df}=49, p<0.05)$.

Given this difference, we assessed the relationship using Pearson product moment correlations between sample size and magnitude of symptom reduction. A statistically significant correlation was found between sample size and symptom reduction among those assigned to antidepressants (both test and comparators, $r=0.23, n=91, p<0.05$ ) as well as those assigned to placebo $(r=0.40, n=51$, $p<0.001$ ).

In order to clarify whether the sample size effect was primarily caused by those assigned to placebo, we conducted an analysis of covariance. We assessed magnitude of symptom reduction as the dependent measure between the flexible and fixed dose trials using sample size as the covariate. This analysis revealed that the difference in placebo response between fixed and flexible dose studies was present even after controlling for sample size $(\mathrm{F}=4.08$, $\mathrm{df}=1,48, p<0.05)$.

The numbers of treatment arms were higher in fixed dose trials, in part because of the nature of these trials. In the 15 fixed dose trials, seven had four or more treatment arms compared to two ( $v s$ placebo alone) or three (when the 
active comparator was also included) in the flexible dose trials. None of these differences reached statistical significance, in part because of large variability.

The number of depressed patients completing the trial was slightly higher in trials with fixed dose compared to flexible dose. Completion rates for those assigned to placebo were $64.7 \%$ in fixed dose trials compared to $57.8 \%$ in flexible dose trials. Completion rates for those assigned to new antidepressants were $63.6 \%$ in fixed dose trials compared to $61.3 \%$ in flexible dose trials. Similarly, completion rates for those assigned to active comparators were $62.5 \%$ in fixed dose trials compared to $61.8 \%$ in flexible dose trials. There were no statistical differences between any of the numeric differences.

We were unable to ascertain the proportion of the depressed patients who prematurely terminated for side effects or lack of effectiveness, because of the nature of FDA SBA reports. (The safety data relating to reasons for termination are calculated and tabulated for all trials and include data from pivotal trials without identifying the latter group.) Most trials in both types of studies were 6-8 weeks long.

Lastly, we assessed if the primary function of the fixed dose studies was achieved among these trials. In other words, we assessed if symptom reduction was larger with higher dose compared to lower doses. Paradoxically, no such relationship was found within the eventual approved dose range (Table 2).

\section{DISCUSSION}

The aim of this analysis was to assess if the dosing schedule in an antidepressant clinical trial affected the outcome of the trial. Our results suggest that the ability to differentiate statistically between test antidepressants and placebo, and active comparator and placebo, is significantly higher in flexible dose trials, compared to fixed dose trials. In fact, the chances of having a positive trial outcome was almost twice as high in flexible dose trials (59.6\%) compared to fixed dose trials $(31.4 \%)$.

This finding, in part, appeared to be related to the outcome with placebo rather than the test or comparator antidepressants $(\mathrm{F}=4.08, \mathrm{df}=1,48, p<0.05)$. A lower magnitude of symptom reduction with placebo was seen in flexible dose trials $(29.3 \%)$ compared to fixed dose trials $(35.8 \%)$. Patient and investigator expectations may contribute to a placebo response. A lower placebo response in flexible dose studies could reflect patient willingness to report ongoing symptoms since the dose can be adjusted. On the other hand, the probability of receiving active treatment (new or comparator) is greater in fixed dose studies, so patients and investigator may assume active treatment is being received.

Of the potential confounding factors that could influence the results, we did not find the presence of active comparator to affect the outcome of trials with both the flexible and fixed dose trials. Duration of clinical trial was similar between flexible and fixed dose trials.

The presence of a higher number of treatment arms in the fixed dose trials did not reach statistical significance, partly because of large variability. Unexpectedly, the number of depressed patients completing the trial when assigned to placebo was numerically higher in fixed dose trials (64.7\%) compared to flexible dose trials (57.8\%) (Khan et al, 2000, 2001). This pattern is in contrast with some of the assumptions about outcome with fixed $v s$ flexible dose trials. Interestingly, the magnitudes of symptom reduction as well as completion rates were similar for antidepressants in both the fixed and flexible dose trials.

The lack of a relationship between dose of the antidepressant and symptom reduction was unexpected, and paradoxically did not provide the answer for which the trials were conducted. Such a finding is not unexpected among psychotropics, although some (Montgomery, 1995; Montgomery et al, 1994) have reported a dose-response relationship. Few caveats that prevent full generalization of this finding are warranted. This lack of a dose-response relationship may be unique to the antidepressants assessed for this study and may not be true for all antidepressants. Also, a dose relationship was found in some of the trials as some 'ineffective' dose data are excluded from this analysis. So, it is reasonable to consider that a threshold effect may occur with these antidepressants rather than a continuing dose-response relationship.

These factors raise the possibility that the flexible dose and fixed dose antidepressant trials are methodologically different. Specifically, factors such as power calculations may need revision, when designing flexible dose trials compared to fixed dose trials.

Although we cannot exclude the possibility that a higher magnitude of side effects may have partially unblinded the investigators in flexible dose trials, our results do not directly support such a possibility. For example, the placebo response difference between trial designs would not likely be influenced by adverse events.

Interestingly, the flexible dose trial scenario is more analogous to clinical practice. The clinician is forced to make a decision as to whether the patient is responding as well as having tolerable side effects and deciding on further dosing. This in turn forces the clinician to decide on the nature of clinical response rather than following a dosing protocol. Alternatively, the clinician is a bystander in fixed dose trials, attempting to make an 'unbiased' judgment about a patient's psychopathological state and is relatively free of forced decision making.

In conclusion, we suggest that the outcome of an antidepressant clinical trial may be influenced by the dosing schedule of the antidepressant. Our results suggest that flexible dosing schedule favors a positive outcome compared to fixed dosing. These findings warrant replication from other independent databases.

\section{REFERENCES}

Benkert O, Szegedi A, Wetzel H (1996). Minimum effective dose for the antidepressants - an obligatory requirement for antidepressant drug evaluation? Int Clin Psychopharmacol 11: 177-185.

Bollini P, Pampallona S, Tibaldi G, Kupelnick B, Munizza C (1999). Effectiveness of antidepressants. Meta-analysis of dose effect relationships in randomized clinical trials. Br J Psychiatry 174: 297-303.

US Congress (1996). Freedom of Information Act. 5 US Congress. 552 (1994 \& Supp. II 1996). Available at http://www.usdoj. 
gov/04foia/. Accessed January 21, 1999. Br J Psychiatry 174: 297-303.

Gram LF (1990). Inadequate dosing and pharmacokinetic variability as confounding factors in assessment of efficacy of antidepressants. Clin Neuropharmacol 3: S35-44.

Khan A, Khan S, Leventhal R, Brown WA (2001). Symptom reduction and suicide risk in patients treated with placebo in antidepressant clinical trials: a replication analysis of the food and drug administration database. Int J Neurosychopharmacol 4: 113-118.

Khan A, Warner HA, Brown WA (2000). Symptom reduction and suicide risk in patients treated with placebo in antidepressant clinical trials: an analysis of the Food and Drug Administration Database. Arch Gen Psychiatry 57: 311-317.

Montgomery SA (1995). Selecting the optimum therapeutic dose of serotonin reuptake inhibitors: studies with citalopram. Int Clin Psychopharmacol 10: 23-27.

Montgomery SA, Pedersen V, Tanghoj P, Rasmussen C, Pioux P (1994). The optimal dosing regimen for citalopram - a metaanalysis of nine placebo-controlled studies. Int Clin Psychopharmacol 9: 35-40.

Schatzberg AF (1991). Dosing strategies for antidepressant agents. J Clin Psychiatry 52: S14029. 